\title{
PEPFAR's Role in Protecting and Leveraging HIV Services in the COVID-19 Response in Africa
}

\author{
Carol W. Holtzman ${ }^{1} \cdot$ Catherine Godfrey $^{2} \cdot$ Lawal Ismail $^{3} \cdot$ Elliot Raizes $^{4} \cdot$ Julie A. Ake $^{5} \cdot$ Fana Tefera $^{6}$. \\ Salome Okutoyi ${ }^{7}$. George K. Siberry ${ }^{8}$
}

Accepted: 8 November 2021 / Published online: 4 January 2022

This is a U.S. government work and not under copyright protection in the U.S.; foreign copyright protection may apply 2022

\begin{abstract}
Purpose of Review We describe the impact of COVID-19 on PEPFAR programs in Africa and how PEPFAR adapted and leveraged its interventions to the changing landscape of the COVID-19 pandemic.

Recent Findings To mitigate the potential impact of COVID-19 on the HIV response and protect the gains, continuity of treatment was the guiding principle regarding the provision of services in PEPFAR-supported countries. As the COVID-19 pandemic matured, PEPFAR's approach evolved from a strictly "protect and salvage" approach to a "restore and accelerate" approach that embraced innovative adaptations in service and "person-centered" care.

Summary The impact of service delivery interruptions caused by COVID-19 on progress towards HIV epidemic control in PEPFAR-supported African countries remains undetermined. With COVID vaccine coverage many months away and more transmissible variants being reported, Africa may experience more pandemic surges. HIV programs will depend on nimble and innovative adaptations in prevention and treatment services in order to advance epidemic control objectives.
\end{abstract}

Keywords PEPFAR $\cdot$ HIV $\cdot$ Africa $\cdot$ COVID-19 $\cdot$ Program adaptation $\cdot$ Continuity of services

This article is part of the Topical Collection on The Global Epidemic

George K. Siberry

gsiberry@usaid.gov

Carol W. Holtzman

choltzman@usaid.gov

Catherine Godfrey

qea0@cdc.gov

Lawal Ismail

ilawal@wrp-n.org

Elliot Raizes

gwq0@cdc.gov

Julie A. Ake

Julie.a.ake.mil@mail.mil

Fana Tefera

obi3@cdc.gov

Salome Okutoyi

sokutoyi@usaid.gov

1 Division of Prevention Care and Treatment, Office of HIV/AIDS Bureau of Global Health United States Agency for International Development, 500 D Street SW, Washington, DC 20547, USA
2 Office of the Global AIDS Coordinator, Department of State, HIV Care and Treatment, PEPFAR, 1800 G St. Ste 10300, DC 20003 Washington, USA

3 Embassy of the United States of America, US Army Medical Research Directorate Africa - Nigeria (USAMRDA-N), Walter Reed Army Institute of Research (WRAIR), CBD, Plot 1075 Diplomatic Drive, Abuja, Nigeria

4 Division of Global HIV/TB (DGHT) Centers for Disease Control and Prevention, GA, Atlanta, USA

5 US Military HIV Research Program, Walter Reed Army Institute of Research, 503 Robert Grant Ave, Silver Spring, MD 20910, USA

6 Centers for Disease Control and Prevention, Ethiopia Entoto Road, Addis Ababa, Ethiopia

7 Health Population and Nutrition Office USAID Kenya and East Africa, 4785-00100 Nairobi, Kenya

8 Division of Prevention Care and Treatment, Office of HIV/AIDS, Bureau of Global Health, United States Agency for International Development, 500 D Street SW, Washington, DC 20547, USA 


\section{Introduction}

There were 22.5 million (uncertainty range $20.1-30.5$ ) people living with HIV (PLHIV) in 2020 in Africa [1]. Africa accounts for nearly one million of the total 1.5 million new cases of HIV globally [1]. The US President's Emergency Plan for AIDS Relief (PEPFAR) supports over 50 countries around the world, including over 25 countries in Africa, to achieve HIV epidemic control, all while significantly strengthening global health security. As of November 8, 2021, WHO reported 6,172,791 confirmed COVID-19 cases and 151,197 deaths in Africa [2]. The COVID-19 pandemic has led to severe disruptions in services for PLHIV, as well as prevention services for those at risk of acquiring HIV. Varying levels of government-containment measures, including lockdowns, and PEPFAR guidance to ensure the safety of healthcare workers and clients have led to the pause and/or scale-down of programmatic activities across the HIV prevention and treatment cascades [ $3 \bullet \bullet$. In addition, access to health facility-based services were interrupted as a result of (1) health facilities repurposed for COVID-19 treatment or closed completely; (2) human resource shortages due to illness, self-quarantine, strikes, or diversion to the COVID-19 response; (3) decreased availability of public transportation for clients; and (4) clients' fear of attending clinic appointments either due to increased risk of COVID19 exposure or police brutality if caught outside during strict lockdown mandates. In several African countries, COVID19 resulted in missed clinic visits and decreases in HIV testing, antiretroviral therapy (ART) initiations, viral load testing, and prevention interventions such as voluntary medical male circumcision (VMMC) and pre-exposure prophylaxis (PrEP) $[4,5 \bullet \bullet, 6,7]$.

To mitigate the potential impact of the COVID-19 pandemic on the HIV response and protect the gains, continuity of treatment became the guiding principle regarding the provision of services in PEPFAR-supported countries [ $3 \bullet \bullet]$. In comparison, HIV prevention services were initially more likely to be dramatically curtailed or even suspended. As the COVID-19 pandemic persisted, PEPFAR's approach evolved from a strictly "protect and salvage" approach to a "restore and accelerate" approach that embraced innovative adaptations in services and "person-centered" care. Here, we describe, with review of available literature, the impact of COVID-19 on PEPFAR programs and how PEPFAR adapted and leveraged its interventions to the changing landscape of the pandemic (Table 1).

\section{HIV Prevention Services}

Many HIV prevention programs were based on community outreach and demand creation that made it difficult to continue during COVID-19 when gatherings were either prohibited or discouraged. There was recognition, however, that PEPFAR needed to weigh the risks versus benefits of limiting or suspending activities that could prevent mortality associated with COVID-19 but result in increased mortality with HIV. According to a modeling study, maintaining VMMC services could lead to an additional 0.004 to 0.24 COVID-19 deaths but may avert 19 - 146 HIVrelated deaths per 10,000 clients $[8 \bullet \bullet]$. Sustained efforts in HIV prevention are crucial to reaching HIV epidemic control. Therefore, PEPFAR placed emphasis on maintaining HIV prevention programs where feasible while adapting COVID-19 prevention measures, promptly resuming programs that were paused, and scaling up biomedical approaches such as PrEP with adaptations.

Many PEPFAR countries prioritized the maintenance and acceleration of PrEP services by ensuring adherence and continuity with existing clients and offering multimonth dispensing (MMD) [9]. Recognizing the need for non-facility-based delivery modalities, South Africa innovated with home and community delivery and online ordering of PrEP, including condoms, lubricants, and self-screening test kits [10]. Lesotho shifted PrEP initiations and refills from large community outreach events to drop-in centers for key and priority populations, while integrating it with other services such as family planning. In Kenya, lubricants and condoms were delivered by community peer educators while observing infection prevention and control measures. Medication-assisted therapy (MAT) services were continued through mobile dispensing and daily access to health facilities, including the provision of passes when inter-county travel was restricted.

VMMC was initially paused but countries gradually resumed services in limited sites after conducting assessments to ensure infection prevention and control (IPC) protocols and other COVID-19 risk mitigation measures were followed. In Lesotho, Large-group demand creation activities (roadshows, public gatherings, door-to-door mobilization) were paused and virtual methods (radio, printed materials, social media) and interpersonal one-onone interactions were employed to generate demand for both PrEP and VMMC, along with provision of COVID-19 prevention messaging and education. 


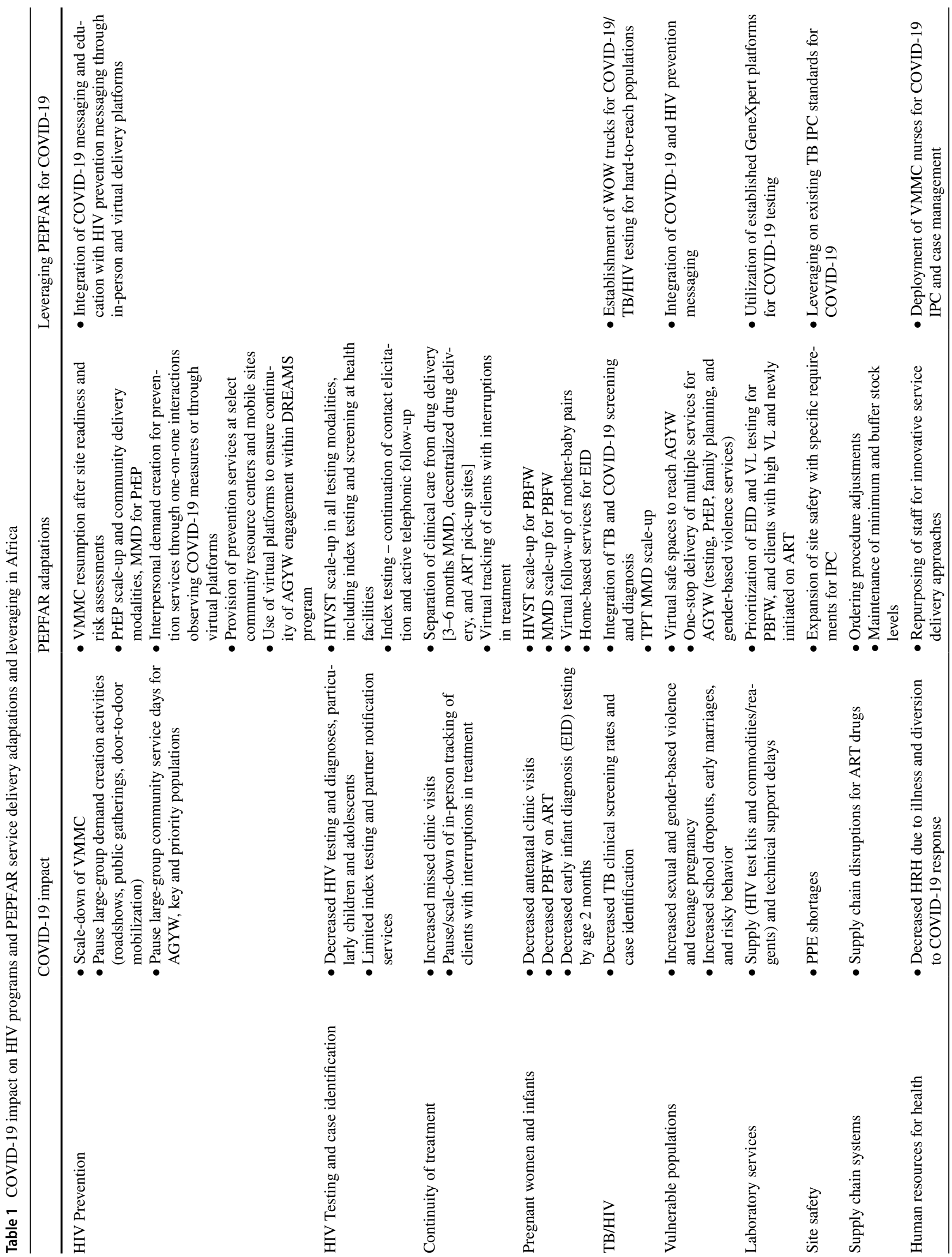




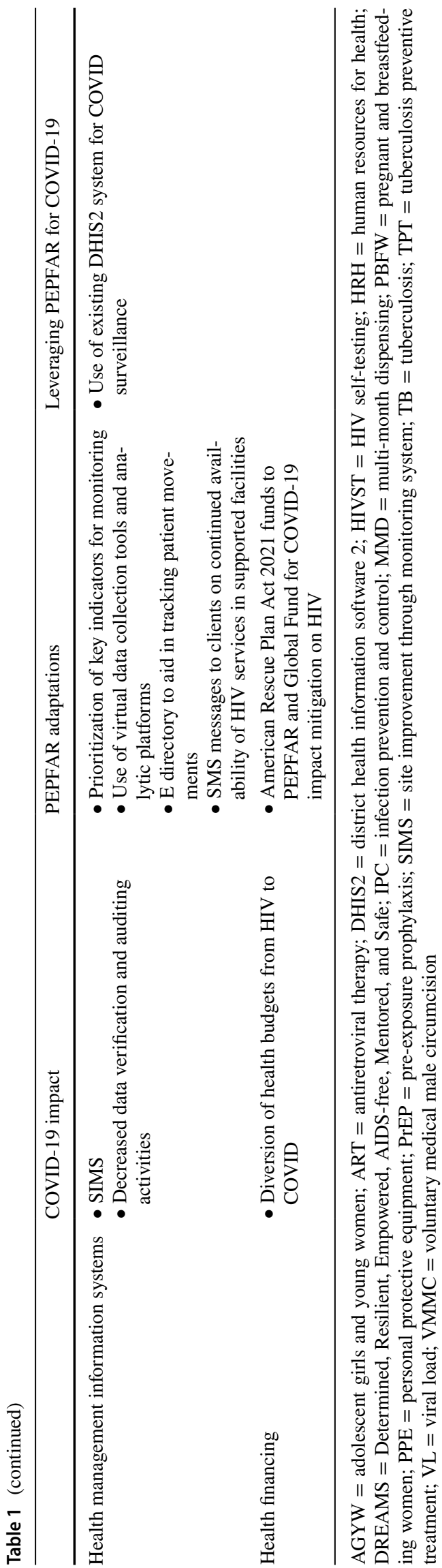

To preserve structural prevention interventions for adolescent girls and young women (AGYW) and orphans and vulnerable children (OVC), countries established active contact with clients through SMS, phone calls, and social media until activities could be safely resumed. In Lesotho, groupbased activities for social asset building or socioeconomic strengthening activities were adapted and conducted outdoors with smaller groups of AGYW using COVID-19 precautions, such as cloth face coverings, physical distancing, and hand hygiene. COVID-19 prevention messaging was integrated into these small group activities at every opportunity. However, most AGYW program curricula were paused and could not be delivered virtually as they had evidencebased skills components dependent on in-person interactions. In Lesotho, post-violence care services were scaled up and gender-based violence (GBV) and "Child-help" hotlines were created or reactivated. School-based HIV and violence prevention and other community mobilization and norms change programs were switched to virtual platforms with curricula delivered through the radio or television.

\section{HIV Testing and Case Identification}

PEPFAR's approach to epidemic control involves aggressive, high-yield HIV case-finding strategies focusing on index testing and partner notification services in health facilities and the community. COVID-19 introduced a variety of barriers to both community- and facility-based testing. Access to HIV testing at health facilities was limited because of site closures, hesitancy to visit health facilities and other reasons. Community outreach and testing services were paused, which eliminated many of the case-finding strategies for key and priority populations. Countries in sub-Saharan Africa experienced significant declines in HIV testing and diagnoses, particularly for children and adolescents, during the COVID-19 pandemic $[5 \bullet \bullet, 11,12]$. However, HIV testing remained crucial as an entry point for prevention, care, and treatment services. In Lesotho, to decongest health facilities and limit contact time with providers, facility-based testing was prioritized for those most in need (e.g., antenatal, tuberculosis (TB), HIV-exposed infants, high-risk patients using a risk screening approach), and HIV self-testing (HIVST) plans were accelerated and scaled up in all testing modalities. Index testing was a critical testing strategy, and PEPFAR encouraged continued contact elicitation and tracking of contacts, but with limitations to virtual methods. These contacts were encouraged to go to health facilities for testing.

Innovative approaches such as HIVST were introduced and scaled up in the PEPFAR programs because they 
offered privacy and convenience and allowed the health care provider to focus on official diagnosis and confirmatory testing [13]. COVID-19 opened the opportunity for countries to institutionalize HIVST for case-finding strategies, including partner-delivered HIVST kits, and to facilitate HIV testing while reducing exposure to COVID-19 in facilities and to staff. In Lesotho, HIVST was rapidly integrated into index testing services and offered to clients to be further distributed to their contacts. For clients visiting health facilities, HIVST was adopted as an additional risk screening approach to enhance targeted case identification. Education and demonstrations were provided outside in a physically-distanced group session, and clients were offered test kits to be self-administered at a booth or at home. In Lesotho, HIVST static pick-up sites and resource centers were established and strategically placed for key and priority populations (e.g., AGYW), and were expanded to include private pharmacies. Demand creation for HIVST was generated through radio, television, and social media.

\section{Continuity of Treatment}

At the beginning of the COVID-19 pandemic, there was concern that PLHIV would have significant disruptions in treatment for a variety of reasons, such as supply chain instability, restricted movements, and fear of exposure to COVID-19 during clinic visits. Given these concerns, the treatment focus of PEPFAR rested squarely on ensuring continuity of care for individuals already on treatment. A critical adaptation was the separation of clinical care from drug delivery which allowed individuals to have the supplies they needed for treatment but provided streamlined access to care when appropriate and necessary. MMD had been a client-centered policy adaptation that PEPFAR advocated, and COVID-19 accelerated the adoption of this policy. COVID-19 guidance recommended that all clients be dispensed at least 3 but preferably 6 months of ART, TB preventive therapy (TPT), and cotrimoxazole therapy [3••]. Eligibility requirements were expanded in many countries and included changes in the requirements for a minimum time on ART, viral suppression, exclusions for pregnancy status, and minimum age requirements. These changes meant that in the fifty PEPFAR-supported countries, MMD increased from being available in $46 \%$ of supported sites in Dec 2019 to $71 \%$ in Dec 2020. The absolute number of clients on MMD nearly doubled from 4.8 million in Dec 2019 to 8.3 million in Dec 2020 [14]. PEPFAR also promoted decentralized drug delivery, in which clients collect their medication at a site away from health facilities, such as a private pharmacy or community center. In South Africa, the proportion of individuals receiving medication from an alternate pick-up site increased from 34 to $50 \%$ shortly after the start of the pandemic [10]. Nigeria implemented home delivery of antiretrovirals ARVs using treatment supporters and peer navigators all aimed at ensuring continuity of treatment. MMD has been shown to improve continuity of treatment, and ongoing efforts are occurring that will document the success of this approach [15]. MMD has been associated with maintained or improved viral suppression (unpublished data from AFRICOS Cohort) [16]. PEPFAR programmatic data across all countries suggest that among individuals who had viral loads measured, viral suppression rates were similar to pre-pandemic rates.

\section{Pregnant Women and Infants}

Services for pregnant, women living with HIV (WLHIV), and their infants were threatened by COVID-19 disruptions in Africa. PEPFAR considered maternal HIV testing and treatment and early infant diagnostic (EID) testing to be essential services $[3 \bullet \bullet$. Modeling projected that mother-to-child HIV transmission rates would increase by more than $60 \%$ in sub-Saharan Africa if COVID-19 interrupted HIV care services for 6 months [17••]. PEPFAR program data across supported countries revealed a drop in mid-2020 in the numbers of women attending antenatal clinics, pregnant WLHIV on ART, and EID testing by age 2 months.

PEPFAR encouraged program adaptations that would minimize contact with the health setting, including shifting these services to community settings or creating separate areas for mother-baby care. As already discussed, HIVST is a key strategy to access testing outside of facilities-including for pregnant and breastfeeding women (PBFW). HIVST for PBFW can be delivered through existing community health workers, mentor-mother groups, and other HIV service platforms. PrEP, a critical HIV prevention method for PBFW at increased risk of HIV infection, was also jeopardized by COVID-19. In South Africa, for instance, PrEP initiation and continuation for PBFW declined significantly during COVID-19 national lockdowns [4]. PBFW had been historically excluded from MMD of ART; in the face of COVID-19 disruptions of facility operations and medication supply chain and as another way to reduce contact with health centers and potential exposure to COVID-19, most countries separated drug delivery from clinical care and permitted MMD access for this population (unpublished PEPFAR survey). PEPFAR encouraged programs to expand phone/SMS support to mothers and infants through existing support mechanisms (e.g., community health workers, peer navigators, mentor mothers) and to identify transport 
methods to bring women or infants who are high-risk or in need of clinical support to the facility. In Nigeria, PEPFAR partners supporting antiretroviral (ARV) prophylaxis and EID testing for HIV-exposed infants recognized that the decline in facility attendance due to COVID-19 was impeding their ability to ensure infants received these services. They adapted their service model so that outreach workers could not only visit homes of exposed infants who missed clinic appointments but also collect dried blood spots in the home for EID. Outreach workers returned results to client homes, and for infants with positive tests, escorted them with their mothers to the health facility for treatment initiation [18].

Innovations and adaptations, like MMD for PBFW and home-based services for EID, have protected essential HIV services for women and children during COVID-19, but they may also be a blueprint for client-centered approaches with improved outcomes thereafter.

\section{TB/HIV}

The COVID-19 pandemic resulted in low TB clinical screening rates and TB case identification [19]. Modeling studies estimated a global decrease in TB case detection by an average of $25 \%$ over a period of 3 months (compared to the level of detection before the pandemic). TB case detection will lead to an estimated additional 190,000 (range $56,000-406,000$ ) TB deaths (a 13\% increase), bringing the total number of TB deaths to 1.66 million (1.3-2.1) in 2020 [20]. COVID-19 total and partial lockdowns disrupted patient access to much-needed health services, including TB/HIV and directly observed therapy services. Access to TB/HIV services was further affected by reluctance of people with potential presumptive TB to seek care for symptoms at health facilities due to COVID-19-related stigma or fear of mandated isolation. PEPFAR encouraged the integration of TB and COVID-19 screening where possible and supported provision of personal protective equipment (PPEs) (face mask, N95, hand sanitizers, etc.) for use by the frontline healthcare workers involved in the TB and COVID-19 diagnosis and management. PEPFAR Nigeria supported the scale-up of a mobile diagnostic facility called "Wellness on Wheel"' (WOW Truck), equipped with a mobile GeneXpert system that had the capacity to test for TB, HIV, and COVID-19 in the hard-to-reach communities. It significantly reduced the turnaround time for test results as the results are released to patients the same day given the high volume of samples collected for processing daily. The truck is equipped with two GeneXpert machines each with four modules and runs an average of 16 samples per day for the 6 to 8 hours of operations. Staff working with the truck were also trained on HIV testing services, COVID-19 IPC orientation and PPEs so that integrated services were provided, and HIV-positive cases were referred to the nearest facility for enrollment. Staff were supported with appropriate PPEs for use during the working hours.

In line with global commitments, PEPFAR programs continued to support and strengthen the implementation of specific TB case detection strategies. Efforts were directed towards early TB case detection among PLHIV by ensuring routine clinical screening using a structured clinical symptom checklist during clinic visits and supporting facilitated referrals for definitive TB diagnosis among patients who screen positive. Other activities across different countries included capacity building for healthcare workers for sputum sample collection, transportation, and results retrieval. PEPFAR Nigeria implemented several strategies to mitigate the effect of COVID-19 on TB case detection, including: site-level implementation of universal safety precautions; routine screening for COVID-19 and TB and bi-directional referrals; and prompt client triaging and service prioritization on symptomatology to strengthen the quality of care. GeneXpert sites were prioritized for PPE, especially N-95 masks respirators since the system was also used for pointof-care testing for COVID-19 across many states in the country. MMD for TPT was scaled up to harmonize with ART drug refills and to prevent multiple clinic visits.

\section{Vulnerable Populations}

With the spread of COVID-19, vulnerable groups including OVC and AGYW and their households were exposed to a significant risk of increased vulnerabilities and poor outcomes. Most vulnerable households were hit hard by forced market closures, movement restrictions, and national curfews as governments put measures in place to control spread of COVID-19. This led to loss of employment due to business closures, rendering many OVC caregivers with reduced income and lacking basic needs and non-HIV health care. Some households were forced to relocate or send children to rural areas to cut down on living costs leading to already vulnerable populations (including children and adolescents living with HIV) missing clinic appointments. Community services targeting these vulnerable populations, including safe spaces and training sessions, halted in accordance with the social distancing measures. The pandemic resulted in increased rates of sexual and gender-based violence and teenage pregnancy due to increased homestay with school closures [21, 22]. In addition, there is evidence of school dropouts, early marriages, and risky behavior, e.g., drug use [23].

The PEPFAR program adapted the use of virtual platforms to reach AGYW through virtual safe spaces. Condoms and family planning (FP) commodities were supplied at 
designated community sites managed by mentors to sustain availability to AGYW. For improved efficiency, access, and reduced contact, PEPFAR encouraged the implementation of a one-stop delivery of multiple services during encounters with AGYW, such as HTS, PrEP, FP, and GBV screening. These adapted HIV services were also leveraged for the COVID-19 response. Some of the COVID-19 mitigation measures implemented for the vulnerable populations include IPC support, IEC material, smaller outdoor group sessions, etc. COVID-19 prevention messaging and community sensitization were integrated with HIV messaging on prevention and treatment and the need for attending antenatal care early in pregnancy.

\section{Laboratory Services}

The impact of COVID-19 on laboratory services was largely driven by supply and demand dynamics. Laboratories experienced delays in receiving HIV test kits and other commodities and reagents because of manufacturing gaps and shipment delays because of air traffic and border closures. In Lesotho, border closures delayed the delivery of spare parts as well as entry of engineers to repair broken instruments, resulting in prolonged down times of viral load platforms. In January 2020, the World Health Organization issued guidance for the use of nucleic acid testing for SARS-CoV-2, which was later updated to include an emergency use listing (EUL) for the use of that technology for diagnostic purposes [24]. PEPFAR recognized the potential impact of increased demand on existing HIV and TB diagnostic networks and developed priorities for viral load testing. Viral loads for pregnant and breastfeeding women, HIV-exposed infants, those with high viral load, and individuals newly initiated on ART were prioritized over routine viral loads for individuals on therapy. PEPFAR-supported testing platforms and laboratory personnel played a key role in rapid implementation of COVID-19 testing at high volumes. Fortunately, the increased demand for COVID-19 diagnostic testing was balanced by decreased demand for viral load testing (decreased phlebotomy due to stable HIV clients avoiding clinics and receiving ART at alternate sites). Despite the decreases in viral load testing, programmatic data suggested that viral suppression was similar to pre-pandemic levels.

\section{Site Safety}

The COVID-19 pandemic highlighted the need for robust infection prevention and control (IPC) systems to protect both clients and staff in health care facilities as well as to maintain continuity of services. PEPFAR has standards for tuberculosis IPC for all sites. However, several programmatic evaluations of adherence to these standards revealed variable implementation of critical elements such as segregation and fast-tracking of individuals with cough and requirement for a focal person tasked with the implementation of TB infection control activities. Programmatic data suggest that larger clinical sites often had these elements in place, while smaller sites did not. PEPFAR guidance for site safety expanded during the pandemic with specific requirements for IPC in the Country Operational Plan 2021 guidance and in regular updates provided to the field $[3 \bullet \bullet, 25]$. Shortages of PPE including medical masks and respirators were an additional challenge, exacerbated by regulatory prohibitions that precluded the use of US Government funds for their procurement. These restrictions were subsequently removed; however, inadequate supply along with inappropriate use of PPE may have contributed to the observed high case rates of COVID-19 among PEPFAR-supported health care workers [26, 27]. In response to the observed need, funds through the American Rescue Plan Act were directed to support a full spectrum of IPC activities at PEPFAR sites, focusing on environmental and administrative controls and essential hygiene infrastructure [28]. Implementation of these activities is expected to begin in the latter part of 2021 .

\section{Supply Chain Systems}

The integrity of the supply chain was compromised in the early part of the pandemic. Drug manufacturing and delivery of raw materials and finished products all experienced delays. The majority of the US Food and Drug Administration-approved ARV manufacturers are based in India, which experienced a $30 \%$ reduction in manufacturing capacity in July 2020 due to COVID-19-related movement restrictions on the workforce. Early in the pandemic, incoming raw materials sourced in China were interrupted due to port closures, and manufacturing was at approximately $30 \%$ capacity. Exportation of pharmaceuticals requires packaging and labeling, typically not produced in the same plants as drugs; however, printing shops and bottle manufacturers experienced reduced capacity because of lockdown restrictions on factory personnel and delayed delivery of these products to the drug manufacturing sites because of limited interstate travel. Within the African continent, significant delays were reported at the port of Mombasa, which is a critical cargo entry port for Indian drugs to most of the African continent. Finally, delays or intermittent closures at international borders were also major contributing factors to disruptions in the supply chain. PEPFAR's response was to change ordering procedures and those related to the maintenance of the minimum and buffer stock levels. The unexpected rapid scale-up of MMD exerted extreme pressure on supply chain systems, which required countries to re-quantify their need, 
change standard operating procedures, place orders much earlier than otherwise planned, and re-renegotiate delivery time. Where sufficient ARV stock was not available for full MMD, countries were instructed to distribute the available drug supply. Buffer stock, available in central warehouses, were moved to facilities where it could be dispensed to clients. Although reductions in buffer stock were reported as shortages, they were part of a deliberate strategy to ensure local access to essential medications.

\section{Human Resources for Health}

The COVID-19 pandemic presented unique challenges to PEPFAR-supported staff needing to provide essential HIV treatment and prevention services while ensuring the safety and well-being of health workers. Staffing and service delivery models, including the use of virtual platforms, were modified to mitigate the impact on progress towards epidemic control while supporting the local COVID19 response as much as possible. With the suspension of non-essential activities, many PEPFAR-supported health care workers were repurposed to participate in innovative approaches to service delivery, such as the introduction or expansion of community-based services and telehealth. Furthermore, critical cadres were redeployed to support COVID-19 case management activities with specific COVID-19 funding through PEPFAR. After the pause in VMMC services, Lesotho quickly trained existing VMMC nurses and placed them at COVID-19 treatment centers to provide HRH surge capacity with a particular focus on screening and triaging for IPC, as well as provide direct care of COVID-19 confirmed and suspect patients admitted to isolation wards, particularly during the initial wave of the pandemic. PEPFAR implementing partners were instructed to support health workers with the tools, airtime, and data required, as well as training and scripts to use available technologies effectively while providing both virtual and/or inperson technical support.

While continuity of essential services in facilities was a high priority, PEPFAR remained committed to supporting the safety of health workers through the implementation of procedures in facilities that minimized the risk of workplace exposures, not only in patient care areas, but also in staff areas. This included universal face coverings and screening for all clients and visitors, continuous medical masking for health care workers, and emphasis on hand hygiene. Failure to fully implement these procedures was associated with facility closures due to reduced staff availability from necessary quarantining of exposed staff. Therefore, ensuring the safety of health workers also enhanced the provision of essential services by reducing or even eliminating clinic closures $[17 \bullet \bullet]$. PEPFAR also provided detailed guidance on training activities, both for facility and communitybased health workers, based on a situational assessment of the COVID-19 epidemiology and any restrictions imposed by the local government. Finally, PEPFAR partners were encouraged to teach skills to increase resilience and conduct routine wellness checks on the health workers they supported to determine their emotional and physical well-being and ability to maintain responsive services. For example, in Kenya the $\mathrm{MOH}$ developed comprehensive guide on mental health and psychosocial support during the COVID-19 pandemic and the Kenya Mental Health Action Plan 2021 -2025 with the aim of supporting the attainment of mental health to the highest standards. PEPFAR through the American Rescue Plan Act has assigned resources towards supporting mental health for both the PLHIVs and the HCWs supporting HIV services.

\section{Health Management Information Systems}

COVID-19 disrupted routine monitoring and reporting of PEPFAR-supported activities, especially for HIV testing, prevention, and treatment. Many countries suspended PEPFAR Site Improvement through Monitoring System (SIMS) in-person site visits, but were allowed to develop virtual approaches to SIMS visits with some restrictions (e.g., prioritizing follow-up versus comprehensive assessments) [29]. Data verification and audits at health facilities were considered non-essential and curtailed to decrease COVID-19 exposure among PEPFAR-supported staff. There was an additional concern that healthcare providers may not routinely document detailed patient information because of limited human resources. In Ethiopia, key indicators were prioritized for monitoring to ensure continuity of treatment with acceptable integrity and quality of data. New virtual data collection tools and analytic platforms were created. Key indicators that tracked 3 and 6 months MMD, loss to follow-up, ART stock, and TPT were uploaded onto mobile devices (online and offline) and reported weekly. Analytic platforms such as Novel Tableau captured data through electronic medical records and from sites that had more than 300 clients on ART. In Lesotho, PEPFAR has strengthened and institutionalized the use of health information systems, such as District Health Information Software 2 (DHIS2) and electronic medical records (eRegisters), in order to improve data quality. DHIS2 was leveraged to assist with COVID-19 vaccine data management and surveillance through newly established modules that enabled health care workers to collect client-level COVID-19 data at all PEPFAR-supported health facilities. In Kenya, the MOH developed an ART e-directory to facilitate tracking and accounting for ART clients receiving ART from their non-primary facility as a result of COVID-19 travel restrictions and also broadcasted 
SMS messages to clients on continued availability of HIV services in all supported facilities.

\section{Health Financing}

The COVID-19 pandemic had a significant impact on donor budgets, as well as those of low- and middle-income countries, which posed risks to HIV program funding. Many donor countries, however, rose to the challenge. The German government made a major EUR 150 million contribution to The Global Fund to Fight AIDS, Tuberculosis, and Malaria (GFATM) in June 2020, early in the coronavirus epidemic, and recently donated another EUR 140 million in support of the ACT-Accelerator program [30]. Through the American Rescue Plan Act (ARPA), PEPFAR was allocated USD 250 million to address the implications of the COVID-19 pandemic for the program [28]. The ARPA also allocated USD 3.5 billion to the GFATM to mitigate the impact of the COVID-19 epidemic in resource-limited settings. In its budget request to the US Congress, the Biden Administration requested additional resources to combat the HIV epidemic domestically as well as globally, including an increase of USD 800 million for global health programming [31].

In low- and middle-income countries, economies were severely curtailed by the pandemic. Income streams were affected by decreased remittances, which were an important source of revenue in these settings [32]. There were drastic impacts on informal employment, which represented $90 \%$ of employment in low-income countries and $67 \%$ of employment in middle-income countries [33]. Debt concerns were mounting as multiple countries in subSaharan Africa, such as Zambia, were under significant financial strain from servicing debt even before the coronavirus pandemic [34]. In this context, domestic investments in health were drained by national COVID-19 response efforts, which further challenged HIV program investments. Circumstances in the Republic of South Africa were illustrative, where over 2.2 million people lost their jobs in the first quarter of the pandemic and the economy retracted by $7 \%$ in 2020 , with growing budget deficits (Department of the National Treasury, South Africa). The National Treasury signaled national spending cuts in the 2021 Medium Term Expenditure Framework period (3 years) totaling USD \$20 billion (of which, USD \$5.3 billion is in the health sector). This could present challenges to meeting increasing demands on HIV and TB grant programs. For PEPFAR, these obstacles were addressed in multi-stakeholder country operational planning processes to ensure continued financing to support the maintenance of critical services for PLHIV.

\section{Conclusion}

The impact of COVID-19 on the HIV response as a result of service delivery interruptions is largely unknown and yet to be determined. Mathematical models have shown that a 6-month interruption in treatment across half the population of PLHIV on ART would lead to 1.63 times (median across models: range 1.39-1.87) increase in HIV-related deaths over a 1-year period compared to no interruptions $[17 \bullet \bullet]$. This would also lead to 1.6 times increase in mother-to-child transmission of HIV [17••]. In another model, if ART treatment is maintained with no new initiations, but VMMC and PrEP interventions cease for 3 months along with condom reductions, HIV deaths would be limited to $<2 \%$ over 5 years [35••]. However, this could lead to a $7 \%$ increase in new HIV infections [35••].

PEPFAR's goal is to ensure the safety of all its beneficiaries and supported staff while continuing to help countries reach HIV epidemic control. Maintaining PLHIV on ART despite disruptions in health service delivery has been PEPFAR's priority since the beginning of the pandemic. It would be important to resume HIV prevention efforts whenever possible to prevent increases in HIV incidence. Unfortunately, COVID-19 will remain a threat to the HIV/TB response in Africa until the pandemic is controlled. Improved COVID vaccine coverage may concomitantly support safer service delivery in health facilities and communities. As of November 8, 2021, only 206 million vaccine doses have been administered in Africa, which represents only about 15 single doses per 100 people, or $2.8 \%$ of the total COVID-19 vaccine doses administered globally [36]. With low vaccine coverage, Africa may experience more pandemic surges and possibly with more transmissible and virulent variants; it would be critical to closely monitor and evaluate HIV/TB programs and adjust interventions and adaptations as necessary. PEPFAR investments are limited to services and activities that directly benefit PLHIV and those at risk for HIV. However, PEPFAR will leverage its investments to the extent possible for health security needs and continue to remain focused on the continuity of services for its beneficiaries.

Funding This article was made possible by the support of the American people through the United States Agency for International Development (USAID), the Center for Disease Control, the United States Army, and the Department of Defense under the U.S. President's Emergency Plan for AIDS Relief (PEPFAR).

\section{Declarations}

Disclaimer The findings and conclusions in this report are those of the authors and do not necessarily represent the official positions of the U.S. Department of State, United States Agency for International 
Development, the Centers for Disease Control and Prevention, the U.S. Army, the Department of Defense, or the U.S. Government.

Conflict of Interest The authors declare no competing interests.

Human and Animal Rights and Informed Consent This article does not contain any studies with human or animal subjects performed by any of the authors.

\section{References}

Papers of particular interest, published recently, have been highlighted as:

- Of importance

$\bullet$ Of major importance

1. Joint United Nations Programme on HIV/AIDS. UNAIDS data 2019. Joint United Nations Programme on HIV/AIDS. 2019. https://aidsinfo.unaids.org/. Accessed 24 May 2021.

2. World Health Organization. WHO coronavirus (COVID-19) dashboard. World Health Organization. 2021.https://covid19. who.int/table. Accessed 24 May 2021.

3. U.S. President's Emergency Plan for AIDS Relief. PEPFAR technical guidance in context of COVID-19 pandemic, May 19, 2021 Update. U.S. Department of State. 2021.https://www. state.gov/wp-content/uploads/2021/05/05.19.21-PEPFARTechnical-Guidance-During-COVID.pdf. Accessed 25 May 2021. This document provides up-to-date and comprehensive PEPFAR technical guidance on how countries should adapt services during the COVID-19 pandemic.

4. Davey DLJ, Bekker LG, Mashele N, Gorbach P, Coates TJ, Myer L. PrEP retention and prescriptions for pregnant women during COVID-19 lockdown in South Africa. Lancet HIV. 2020;7(11):e735.

5. J Dorward T Khubone K Gate H Ngobese Y Sookrajh S Mkhize et al 2021 The impact of the COVID-19 lockdown on HIV care in 65 South African primary care clinics: an interrupted time series analysis Lancet HIV 83 e158 e165. This interrupted time series analysis in South Africa showed that the 2020 national COVID-19 lockdown resulted in dramatic decreases in HIV testing and ART initiations in KwaZuluNatal province.

6. Mukwenha S, Dzinamarira T, Mugurungi O, Musuka G. Maintaining robust HIV and tuberculosis services in the COVID-19 era: a public health dilemma in Zimbabwe. Int J Infect Dis. 2020;100:394-5.

7. Joint United Nations Programme on HIV/AIDS. COVID -19's HIV vertical transmission services reversed, Oct. 27, 2020. Joint United Nations Programme on HIV/AIDS. 2020. https:// www.unaids.org/en/resources/presscentre/featurestories/2020/ october/20201027_covid19-impact-hiv-vertical-transmission. Accessed 24 May 2021.

8. Stover J, Kelly SL, Mudimu E, Green D, Smith T, Taramusi I, et al. The risks and benefits of providing HIV Services during the COVID-19 pandemic. medRxiv. 2021. https://doi.org/10. $1101 / 2021.03 .01 .21252663$. This modeling study examined the trade-offs of COVID-related population movement restrictions and health service limitations with regards to SAR-CoV-2 and HIV mortality. Maintaining HIV services could lead to an additional 0.002 to 0.15 per 10,000
COVID-19 deaths, while limiting such services could lead to an additional 19-146 per 10,000 HIV-related deaths.

9. Mahwire TC, Koloane N, Burgess J, Makaba Z, Serrao C, Malone TM. Upscaling HIV pre-exposure prophylaxis implementation during COVID-19 pandemic. Conference on Retroviruses and Opportunistic Infections. June 3 - November 3, 2021. https://www.croiconference.org/abstract/upscalinghiv-preexposure-prophylaxis-implementation-during-covid19-pandemic/. Accessed 12 May 2021.

10. Liu L, Christie S, Munsamy M, Roberts P, Pillay M, Shenoi SV, et al. Title: Expansion of a national differentiated service delivery model to support people living with HIV and other chronic conditions in South Africa: a descriptive analysis. BMC Health Serv Res. 2021;21(1):463.

11. Gross J, Hrapcak S, Rivadeneira E, Svrivastava M, Grillo M, Gurpreet K, et al. Pediatric and adolescent HIV testing and diagnosis in the context of COVID-19. Conference on Retroviruses and Opportunistic Infections. June 3 - November 3, 2021. https:// www.croiconference.org/abstract/pediatric-and-adolescent-hivtesting-and-diagnosis-in-the-context-of-covid-19/. Accessed 12 May 2021.

12. Drammeh B, Dee A, Lasry A, Medley A, Aholou T, Yee R, et al. Changes in HIV testing services after COVID-19 in 11 sub-Saharan African countries. Conference on Retroviruses and Opportunistic Infections. June 3 - November 3, 2021. https:// www.croiconference.org/abstract/changes-in-hiv-testing-servi ces-after-covid-19-in-11-sub-saharan-african-countries/. Accessed 12 May 2021.

13. Ortblad KF, Stekler JD. HIV self-testing: finding its way in the prevention tool box. BMC Med. 2020;18(1):373.

14. Bailey LS, Agaba P, Douglas M, Clinkscales J, Godfrey C. COVID-19 catalyzed expanded multi-month dispensing policies for antiretroviral therapy (ART), increasing coverage in PEPFAR-supported countries and accelerating expectations for the future of client-centered differentiated care. J Int AIDS Soc. 2021. In Press.

15. Fatti G, Ngorima-Mabhena N, Mothibi E, Muzenda T, Choto R, Kasu T, et al. Outcomes of three- versus six-monthly dispensing of antiretroviral treatment (ART) for stable HIV patients in community ART refill groups: a cluster-randomized trial in Zimbabwe. J Acquir Immune Defic Syndr. 2020;84(2):162-72.

16. Kim MH, Wanless RS, Caviness AC, Golin R, Amzel A, Ahmed $\mathrm{S}$, et al. Multimonth prescription of antiretroviral therapy among children and adolescents: experiences from the Baylor International Pediatric AIDS Initiative in 6 African Countries. J Acquir Immune Defic Syndr. 2018;78(Suppl 2):S71-80.

17. BL Jewell E Mudimu J Stover D Brink Ten AN Phillips JA Smith et al 2020 Potential effects of disruption to HIV programmes in sub-Saharan Africa caused by COVID-19: results from multiple mathematical models Lancet HIV 79 e629 e640 . This modeling study showed that a 6-month interruption in treatment across half the population of PLHIV on ART would lead to 1.63 times increase in HIV-related deaths over a 1-year period compared to no interruptions.

18. Fadare O, Emerenini F, Yakubu T, Dare B, Ijaiya M, Ogundare Y, et al. Sustaining early infant diagnosis of HIV during COVID19 pandemic: experience collecting dry blood spots samples at home from HIV-exposed infants in Nigeria. Pediatr Infect Dis J. 2021;40(12):e529-30.

19. Odume B, Falokun V, Chukwuogo O, Ogbudebe C, Useni S, Nwokoye N, et al. Impact of COVID-19 on TB active case finding in Nigeria. Public Health Action. 2020;10(4):157-62.

20. Graziou P. Predicted impact of the COVID-19 pandemic on global tuberculosis deaths in 2020. medRxiv. 2021; https://doi. org/10.1101/2020.04.28.20079582. 
21. United Nations Children's Fund. Responding to the shadow pandemic: taking stock of gender-based violence risks and responses during COVID-19, August 20, 2020. United Nations Children's Fund. 2020. https://www.unicef.org/media/76916/file/GenderBased-Violence-in-Emergencies-CP-Learning-Brief-Aug-2020. pdf. Accessed 27 May 2021.

22. United Nations Children's Fund. COVID-19 - GBV risks to adolescent girls and interventions to protect and empower them. United Nations Children's Fund. 2020. https://www.unicef.org/ media/68706/file/COVID-19-GBV-risks-to-adolescent-girlsand-interventions-to-protect-them-2020.pdf. Accessed 27 May 2021.

23. United Nations Children's Fund. Getting girls back to the classroom after COVID-19 school closures. United Nations Children's Fund. 2021. https://www.unicef.org/media/94931/file/ Getting\%20girls\%20back\%20to\%20the\%20classroom\%20after\% 20COVID-19\%20school\%20closures\%20(South\%20Sudan).pdf. Accessed 27 May 2021.

24. World Health Organization. Laboratory testing for coronavirus disease (COVID-19) in suspected human cases: interim guidance, 19 March 2020. World Health Organization. 2020. https:// apps.who.int/iris/handle/10665/331501. Accessed 19 May 2021.

25. U.S. President's Emergency Plan for AIDS Relief. PEPFAR 2021 country and regional operational plan (COP/ROP) guidance for all PEPFAR Countries. U.S. Department of State. 2021. https:// www.state.gov/wp-content/uploads/2021/02/PEPFAR-COP21Guidance-Final.pdf. Accessed 25 May 2021.

26. Nunes MC, Baillie VL, Kwatra G, Bhikha S, Verwey C, Menezes $\mathrm{C}$, et al. SARS-CoV-2 infection among healthcare workers in South Africa: a longitudinal cohort study. Clin Infect Dis. 2021.

27. Atnafie SA, Anteneh DA, Yimenu DK, Kifle ZD. Assessment of exposure risks to COVID-19 among frontline health care workers in Amhara Region, Ethiopia: a cross-sectional survey. PLoS One. 2021;16(4):e0251000.

28. 117th United States Congress. American rescue plan act 2021. 117th United States Congress. 2021. https://www.congress. gov/bill/117th-congress/house-bill/1319/text. Accessed 22 May 2021.

29. U.S. President's Emergency Plan for AIDS Relief. Site improvement through monitoring systems (SIMS) Version 4.1, March 8, 2021. U.S. Department of State. 2021. https://datim.zende
sk.com/hc/en-us/sections/200929305-SIMS. Accessed 25 May 2021.

30. The Global Fund to Fight AIDS, Tuberculosis and Malaria. Global Fund thanks Germany for EUR 140 million contribution, April 8, 2021. The Global Fund to Fight AIDS, Tuberculosis and Malaria. 2021. https://www.theglobalfund.org/en/news/2021-0408-global-fund-thanks-germany-for-eur-140-million-contributi on/. Accessed 21 May 2021.

31. The White House. Summary of the President's discretionary funding request, April 9, 2021. The White House. 2021. https:// www.whitehouse.gov/wp-content/uploads/2021/04/FY2022Discretionary-Request.pdf. Accessed 22 May 2021.

32. Joint United Nations Programme on HIV/AIDS. COVID-19 and HIV: Progress report of 2020, Nov. 23, 2020. Joint United Nations Programme on HIV/AIDS. 2020. https://www.unaids. org/sites/default/files/media_asset/COVID-19_HIV_EN.pdf. Accessed 22 May 2021.

33. United Nations. Policy brief: COVID-19 in an urban world, July 2020. United Nations. 2020. https://www.un.org/sites/un2.un. org/files/sg_policy_brief_covid_urban_world_july_2020.pdf. Accessed 20 May 2021.

34. Ravelo JL. Unlocking HIV/AIDS financing during COVID-19, July 6, 2020. Devex. 2020. https://www.devex.com/news/unloc king-hiv-aids-financing-during-covid-19-97633. Accessed 20 May 2021.

35. Jewell BL, Smith JA, Hallett TB. Understanding the impact of interruptions to HIV services during the COVID-19 pandemic: a modelling study. EClinicalMedicine. 2020;26:100483 This modeling study showed that if VMMC and PrEP interventions cease for 3 months along with condom reductions, there would be a seven percent increase in new HIV infections.

36. Ritchie H, Ortiz-Ospina E, Beltekian D, Mathieu E, Hasell J, Macdonald B, et al. Our world in data COVID-19 dataset. 2021. https://ourworldindata.org/covid-vaccinations. Accessed 24 May 2021.

Publisher's Note Springer Nature remains neutral with regard to jurisdictional claims in published maps and institutional affiliations. 